\title{
JEAN-LOUIS NICOLAS
}

\section{VARANASI SITARAMAIAH}

\section{On a class of $\psi$-convolutions characterized by the identical equation}

Journal de Théorie des Nombres de Bordeaux, tome 14, $\mathrm{n}^{\circ} 2$ (2002), p. 561-583

<http://www.numdam.org/item?id=JTNB_2002_14_2_561_0>

(C) Université Bordeaux 1, 2002, tous droits réservés.

L'accès aux archives de la revue "Journal de Théorie des Nombres de Bordeaux » (http://jtnb.cedram.org/) implique l'accord avec les conditions générales d'utilisation (http://www.numdam.org/conditions). Toute utilisation commerciale ou impression systématique est constitutive d'une infraction pénale. Toute copie ou impression de ce fichier doit contenir la présente mention de copyright.

\section{Numdam}

Article numérisé dans le cadre du programme

Numérisation de documents anciens mathématiques

http://www.numdam.org/ 


\title{
On a class of $\psi$-convolutions characterized by the identical equation
}

\author{
par JEAN-Louis NICOLAS et VARANASI SITARAMAIAH*
}

dedicated to Michel Mendès France for his 60th birthday

\begin{abstract}
RÉsumÉ. Dans le cadre de la convolution de Dirichlet des fonctions arithmétiques, R. Vaidyanathaswamy a obtenu en 1931 une formule de calcul de $f(m n)$ valable pour toute fonction multiplicative $f$ et tout couple d'entiers positifs $m$ et $n$. Dans [7], cette formule a été généralisée aux $\psi$-convolutions appelées convolutions de Lehmer-Narkiewicz, qui, entre autres, conservent la multiplicativité. Dans cet article, nous démontrons la réciproque.

ABSTRACT. The identical equation for multiplicative functions established by $R$. Vaidyanathaswamy in the case of Dirichlet convolution in 1931 has been generalized to multiplicativity preserving $\psi$-convolutions satisfying certain conditions (cf. [7]) which can be called as Lehmer-Narkiewicz convolutions for some reasons. In this paper we prove the converse.
\end{abstract}

\section{Introduction}

In 1930, R. Vaidyanathaswamy (see [9] and [10, Section VI]) established the following remarkable identity valid for any multiplicative function and known as the identical equation for multiplicative functions. If $f$ is any multiplicative function, then for any positive integers $m$ and $n$, we have

$$
f(m n)=\sum_{\substack{a|m \\ b| n}} f(m / a) f(n / b) f^{-1}(a b) G(a, b),
$$

where $f^{-1}$ is the inverse of $f$ with respect to the familiar Dirichlet convolution so that

$$
\sum_{d \mid m} f(d) f^{-1}(m / d)=e(m)
$$

Manuscrit reçu le 11 mai 2000.

* Research partially supported by CNRS, Institut Girard Desargues, UMR 5028 and IndoFrench cooperation in Mathematics. 
for all positive integers $m$, where

$$
e(m)= \begin{cases}1, & \text { if } m=1 \\ 0, & \text { if } m>1\end{cases}
$$

and

$$
G(a, b)=\left\{\begin{array}{l}
(-1)^{\omega(a)}, \quad \text { if } r(a)=r(b) \\
0, \quad \text { otherwise, }
\end{array}\right.
$$

$\omega(a)$ being the number of distinct prime factors of $a$ and $r(a)$ the product of distinct prime factors of $a$ with $\omega(1)=0$ and $r(1)=1$.

In [7] the identical equation (1.1) has been generalized to a class of $\psi$ convolutions (which are certain binary operations in the set of arithmetic functions introduced by D.H. Lehmer [2]): if $(m, n) \in T$, where $T$ is the domain of $\psi$, then for any multiplicative function $f$,

$$
f(\psi(m, n))=\sum_{\substack{a, x, b, y \\ \psi(a, x)=m \\ \psi(b, y)=n}} f(x) f(y) f^{-1}(\psi(a, b)) G(a, b)
$$

$f^{-1}$ being the inverse of $f$ with respect to $\psi$ (Section 2 contains undefined notions used in this section); this class of $\psi$-convolutions in which (1.4) has been established is contained in the class of $\psi$-convolutions preserving multiplicativity and satisfying $\psi(x, y) \geq \max \{x, y\}$ for $x, y \in T$, as subclass: in addition to these, they satisfy certain other properties similar to those of regular $A$-convolutions [3]. It is interesting to note that these convolutions have been subsequently characterized (see [8], Corollary 4.1; also see $\S 2$ of the present paper) and have been named [8] as Lehmer-Narkiewicz convolutions. Thus (1.4) holds when $\psi$ is a Lehmer-Narkiewicz convolution.

The object of the present paper is to prove the converse. We show that (see Sections 3, 4 and 5) if $\psi$ preserves multiplicativity, $\psi(x, y) \geq \max \{x, y\}$ for all $x, y \in T$ and the identity (1.4) holds for all multiplicative functions, then $\psi$ is a Lehmer-Narkiewicz convolution; in particular, if $T=\mathbb{Z}^{+} \times \mathbb{Z}^{+}$, $\mathbb{Z}^{+}$being the set of positive integers, it follows from Corollary 4.1 in [8] (also, see Section 2, Lemma 2.16) that $\psi$ is a Dirichlet convolution. Thus the only $\psi$-convolution with domain $T=\mathbb{Z}^{+} \times \mathbb{Z}^{+}$, preserving multiplicativity, satisfying $\psi(x, y) \geq \max \{x, y\}$ for all $x, y \in T$ and with respect to which (1.4) holds for all multiplicative functions, is the Dirichlet convolution.

$\S 2$ deals with preliminaries. Sections 3-5 contain the main results.

For a brief discussion on different proofs and generalizations of the identical equation (1.1) and for certain special cases of (1.4), we refer to [7].

We are pleased to thank the referee for suggesting several improvements on the presentation of our paper. 


\section{Preliminaries}

Let $\emptyset \neq T \subseteq \mathbb{Z}^{+} \times \mathbb{Z}^{+}$and $\psi: T \longrightarrow \mathbb{Z}^{+}$be a mapping satisfying the following conditions (here $\mathbb{Z}^{+}$denotes the set of positive integers):

For each $n \in \mathbb{Z}^{+}, \psi(x, y)=n$ has a finite number of solutions.

$$
\text { If }(x, y) \in T \text {, then }(y, x) \in T \text { and } \psi(x, y)=\psi(y, x) .
$$

$$
\left\{\begin{array}{l}
\text { The statements " }(x, y) \in T,(\psi(x, y), z) \in T " \\
\text { and " }(y, z) \in T,(x, \psi(y, z)) \in T \text { " are equivalent; if one of these } \\
\text { conditions holds, we have } \psi(\psi(x, y), z)=\psi(x, \psi(y, z))
\end{array}\right.
$$

Let $F$ denote the set of arithmetic functions (i.e. complex valued functions defined on $\mathbb{Z}^{+}$). If $f, g \in F$ then the $\psi$-product of $f$ and $g$ denoted by $f \psi g$ is defined by

$$
(f \psi g)(n)=\sum_{\psi(x, y)=n} f(x) g(y)
$$

for all $n \in \mathbb{Z}^{+}$.

The concept of $\psi$-convolution given in (2.4) is due to D.H. Lehmer [2]. It is easily seen that $(F,+, \psi)$ is a commutative ring. Let $\psi(x, y)=x y$ for all $(x, y) \in T$. If $T=\mathbb{Z}^{+} \times \mathbb{Z}^{+}$then $\psi$ in (2.4) reduces to the Dirichlet convolution. If $T=\left\{(x, y) \in \mathbb{Z}^{+} \times \mathbb{Z}^{+}:(x, y)=1\right\}$, then $\psi$ reduces to the unitary convolution [1]. More generally, if $T=\bigcup_{n=1}^{\infty}\{(d, n / d): d \in A(n)\}$, where $A$ is Narkiewicz's regular convolution [3], then $\psi$ reduces to the $A$ convolution. Thus the binary operation in (2.4) is more general than that of Narkiewicz's $A$-convolution.

The following results (Lemmas 2.5 and 2.6) describe necessary and sufficient conditions concerning the existence of unity and inverses in $(F,+, \psi)$.

Lemma 2.5 (cf. [5, Theorem 2.2]). Let $(F,+, \psi)$ be a commutative ring and $\psi(x, y) \geq \max \{x, y\}$ for all $x, y \in T$. Then $(F,+, \psi)$ possesses the unity if and only if for each $k \in \mathbb{Z}^{+}, \psi(x, k)=k$ has a solution. In such a case if $g$ stands for the unity, then for each $k \in \mathbb{Z}^{+}$,

$$
g(k)=\left\{\begin{array}{l}
1-\sum_{\psi(x, k)=k} g(x), \quad \text { if } \psi(k, k)=k \\
0 \quad \text { if } \psi(k, k) \neq k
\end{array}\right.
$$

Lemma 2.6 (cf. [4], also see [10, Remark 1.1]). Let $\psi$ satisfy (2.1) (2.3) and $\psi(x, y) \geq \max \{x, y\}$ for all $x, y \in T$. For each $k \in \mathbb{Z}^{+}$, let the equation $\psi(x, k)=k$ have a solution so that the unity exists in $(F,+, \psi)$. 
Let $g$ denote the unity. Then $f \in F$ is invertible with respect to $\psi$ if and only if

$$
S_{f}(k) \stackrel{\text { def }}{=} \sum_{\psi(x, k)=k} f(x) \neq 0
$$

for all $k \in \mathbb{Z}^{+}$. In such a case, this inverse denoted by $f^{-1}(k)$ can be computed by

and for $k>1$,

$$
f^{-1}(1)=\frac{1}{f(1)}
$$

$$
f^{-1}(k)=\left(S_{f}(k)\right)^{-1}\left[g(k)-\sum_{\substack{\psi(x, y)=k \\ y<k}} f(x) f^{-1}(y)\right] .
$$

The binary operation $\psi$ in (2.4) is called multiplicativity preserving (cf. [6]) if $f \psi g$ is multiplicative whenever $f$ and $g$ are ; as usual, $f \in F$ is called multiplicative if $f(1)=1$ and $f(m n)=f(m) f(n)$ whenever $(m, n)=1$.

The following results (Lemmas 2.7 and 2.9) give a characterization of multiplicativity preserving $\psi$-functions satisfying (i) $\psi(x, y) \geq \max \{x, y\}$ for all $x, y \in T$ and (ii) $\psi(1, k)=k$ for all $k \in \mathbb{Z}^{+}$:

Lemma 2.7 (cf. [6, Theorem 3.1]). Let $\psi$ satisfy $(2.1)-(2.3), \psi(x, y) \geq$ $\max \{x, y\}$ for all $x, y \in T$ and $\psi(1, k)=k$ for all $k \in \mathbb{Z}^{+}$. Suppose that the binary operation $\psi$ in (2.4) is multiplicativity preserving. If $x=\prod_{i=1}^{r} p_{i}^{\alpha_{i}}$ and $y=\prod_{i=1}^{r} p_{i}^{\beta_{i}}$, where $p_{1}, p_{2}, \ldots, p_{r}$ are distinct primes, $\alpha_{i}$ and $\beta_{i}$ are non-negative integers, we have

(a) $(x, y) \in T$ if and only if $\left(p_{i}^{\alpha_{i}}, p_{i}^{\beta_{i}}\right) \in T$ for $i=1,2, \ldots, r$.

(b) For each prime $p$ and non-negative integers $\alpha, \beta$ such that $\left(p^{\alpha}, p^{\beta}\right) \in$ $T$, there is a unique non-negative integer $\theta_{p}(\alpha, \beta) \geq \max \{\alpha, \beta\}$ such that $\psi\left(p^{\alpha}, p^{\beta}\right)=p^{\theta_{p}(\alpha, \beta)}$.

(c) If $(x, y) \in T$, then

$$
\psi(x, y)=\prod_{i=1}^{r} p_{i}^{\theta_{p_{i}}\left(\alpha_{i}, \beta_{i}\right)}
$$

Lemma 2.9 (cf. [6, Theorem 3.2]). Let $T \subseteq \mathbb{Z}^{+} \times \mathbb{Z}^{+}$be such that

(a) $(1, x) \in T$ for every $x \in \mathbb{Z}^{+}$.

(b) $(x, y) \in T$ if and only if $(y, x) \in T$.

(c) If $x$ and $y$ are as given in Lemma 2.7, then $(x, y) \in T$ if and only if $\left(p_{i}^{\alpha_{i}}, p_{i}^{\beta_{i}}\right) \in T$ for $i=1,2, \ldots, r$.

Further, for each prime $p$ and non-negative integers $\alpha, \beta$ such that $\left(p^{\alpha}, p^{\beta}\right) \in T$, let $\theta_{p}(\alpha, \beta)$ be a non-negative integer satisfying 
(d) $\theta_{p}(\alpha, \beta) \geq \max \{\alpha, \beta\}$

(e) $\theta_{p}(\alpha, \beta)=0$ if and only if $\alpha=\beta=0$

(f) $\theta_{p}(0, \alpha)=\alpha$ for every $\alpha \geq 0$

(g) $\theta_{p}(\alpha, \beta)=\theta_{p}(\beta, \alpha)$

(h) For non-negative integers $\alpha, \beta, \gamma$ and for any prime $p$, the statements " $\left(p^{\beta}, p^{\gamma}\right) \in T,\left(p^{\alpha}, p^{\theta_{p}(\beta, \gamma)}\right) \in T$ " and $\left(p^{\alpha}, p^{\beta}\right) \in T$ and $\left(p^{\theta_{p}(\beta, \gamma)}, p^{\gamma}\right) \in T$ " are equivalent; when one of these conditions holds, we have

$$
\theta_{p}\left(\alpha, \theta_{p}(\beta, \gamma)\right)=\theta_{p}\left(\theta_{p}(\alpha, \beta), \gamma\right)
$$

If for $(x, y) \in T, \psi(x, y)$ is defined by $(2.8)$, then $(F,+, \psi)$ is a commutative ring with unity $e$, where $e$ is given by (1.2). Also, f $\psi g$ is multiplicative whenever $f$ and $g$ are.

Theorem 2.10 (cf. [7, Theorem]). Let T, $\psi$ and $\theta_{p}$ be as in Lemma 2.9. Further we assume that for each prime $p$, we have

$$
\begin{gathered}
\left(\theta_{p}(\alpha, \beta)=\theta_{p}(\alpha, \gamma)\right) \Rightarrow(\beta=\gamma) \\
\left(\theta_{p}(\alpha, \beta)=\theta_{p}(\gamma, \delta)\right) \Rightarrow\left\{\begin{array}{l}
\alpha=\theta_{p}(\gamma, c) \text { for some } c \geq 0 \\
\text { or } \beta=\theta_{p}(\delta, d) \text { for some } d \geq 0
\end{array}\right.
\end{gathered}
$$

If $f$ is multiplicative, then the identity in (1.4) holds.

Definition 2.13. Let $\psi$ be multiplicativity preserving with $\psi(x, y) \geq$ $\max \{x, y\}$ for all $(x, y) \in T$ and $\psi(1, k)=k$ for all $k \in \mathbb{Z}^{+}$. Let $T$ and $\theta_{p}$ as in Lemma 2.9. Then $\psi$ is called a Lehmer-Narkiewicz convolution or simply an L-N convolution if $\theta_{p}$ satisfies (2.11) and (2.12) for all primes $p$.

Definition 2.14 (see [3]). A binary operation $B$ in the set of arithmetic functions $F$ is called a regular convolution if

(a) $(F,+, B)$ is a commutative ring with unity.

(b) $B$ is multiplicativity preserving i.e. $f B g$ is multiplicative whenever $f$ and $g$ are.

(c) The constant function $1 \in F$ has an inverse which is 0 or -1 at prime powers.

For each positive integer $n$, if $A(n)$ is a non-empty subset of divisors of $n$, Narkiewicz [3] defines the binary operation $A$ in $F$ (called the $A$ convolution) by

$$
(f A g)(n)=\sum_{d \in A(n)} f(d) g(n / d)
$$

for all $n \in \mathbb{Z}^{+}$. He then calls the convolution $A$ given in (2.15) as regular if $A$ satisfies the definition (2.14). To make a distinction between a general regular convolution as defined in (2.14) and that of the special operation 
$A$ defined in (2.15) which is regular, we call the later as regular Narkiewicz convolution.

The following result gives a characterization of L-N convolutions :

Lemma 2.16 (cf. [8, Corollary 4.1]). For each prime $p$, let $\pi_{p}$ denote a class of subsets of non-negative integers such that

(i) the union of all members of $\pi_{p}$ is the set of non-negative integers.

(ii) each member of $\pi_{p}$ contains zero.

(iii) no two members of $\pi_{p}$ contain a positive integer in common.

If $S \in \pi_{p}$ and $S=\left\{a_{0}, a_{1}, a_{2}, \ldots\right\}$ with $0=a_{0}<a_{1}<a_{2}<\cdots$, we define $\theta_{p}\left(a_{i}, a_{j}\right)=a_{i+j}$, if $a_{i}, a_{j}$ and $a_{i+j} \in S$ ( $i$ and $j$ need not to be distinct). If $\psi$ and $T$ are as given in Lemma 2.9 then $\psi$ is an $L-N$ convolution and is also a regular convolution. Also, every $L-N$ convolution can be obtained in this way.

It is clear from the above result that there are infinitely many L-N convolutions. If $\theta_{p}(x, y)=x+y$, for all $x, y$ such that $\left(p^{x}, p^{y}\right) \in T$, then $\pi_{p}$ should consist of arithmetic progressions. Thus, Lemma 2.16 reduces to the characterization Theorem on regular Narkiewicz's convolutions (cf. [3], Theorem II).

It is interesting to note that a Lehmer-Narkiewicz convolution $\psi$ with domain $T=\mathbb{Z}^{+} \times \mathbb{Z}^{+}$is the Dirichlet convolution. For, if $T=\mathbb{Z}^{+} \times \mathbb{Z}^{+}$, the domain of $\theta_{p}$ in Lemma 2.16 is $\left(\mathbb{Z}^{+} \cup\{0\}\right) \times\left(\mathbb{Z}^{+} \cup\{0\}\right)$. Hence there can be only one member $S$ of $\pi_{p}$ viz: $S=\{0,1,2, \ldots\}$ and $\theta_{p}(i, j)=i+j$ for all non-negative integers $i$ and $j$. Hence $\psi(x, y)=x y$ for all positive integers $x$ and $y$ so that $\psi$ is the Dirichlet convolution.

Lemma 2.17. Let $\psi$ be as in Lemma 2.6. Then the following statements are equivalent:

(a) Every multiplicative function is invertible with respect to $\psi$.

(b) For each $k \in \mathbb{Z}^{+}, \psi(x, k)=k$ if and only if $x=1$.

(Thus, if (a) holds $e$ is the unity in $(F,+, \psi)$ ).

Proof. We assume (a). Since $e$ (given in (1.2)) is multiplicative and is invertible with respect to $\psi$ by hypothesis, $S_{e}(k) \neq 0$ for all $k \in \mathbb{Z}^{+}$by Lemma 2.6. Since

$$
0 \neq S_{e}(k)=\sum_{\psi(x, k)=k} e(x)=\sum_{\psi(1, k)=k} 1,
$$

it is clear that $(1, k) \in T$ and $\psi(1, k)=k$. We now show that $\psi(x, k)=k$ implies $x=1$. This being true when $k=1$, we may assume that $k>1$. Assume that we can find $x_{0}>1$ such that $\psi\left(x_{0}, k\right)=k$. Let $1<x_{0}<$ $x_{1}<\cdots<x_{r}$ be all the solutions of $\psi(x, k)=k$ (from (2.1), there are only finitely many solutions). Let

$$
x_{0}=p_{1}^{\alpha_{1}} \ldots p_{t}^{\alpha_{t}}
$$


where $p_{1}, \ldots, p_{t}$ are distinct primes, $\alpha_{1}, \alpha_{2}, \ldots, \alpha_{t}$ are positive integers and, since $x_{0}>1, t$ is positive. Let $S$ denote the set of prime powers $p_{1}^{\alpha_{1}}, \ldots, p_{t}^{\alpha_{t}}$. We define the multiplicative function $f$ by $f(1)=1, f\left(p_{1}^{\alpha_{1}}\right)=-1$ and

$$
f\left(p^{x}\right)= \begin{cases}1, & \text { if } p^{x} \in S-\left\{p_{1}^{\alpha_{1}}\right\} \\ 0, & \text { if } 1<p^{x} \notin S .\end{cases}
$$

Clearly $f\left(x_{0}\right)=-1$. Fix $j, 1 \leq j \leq r$. We can find a prime power $q^{\beta}>1$ such that $q^{\beta} \| x_{j}$ and $q^{\beta} \notin S$. Hence $f\left(x_{j}\right)=0$. This is true for $j=1,2, \ldots, r$. Thus

$$
\begin{aligned}
S_{f}(k) & =\sum_{\psi(x, k)=k} f(x)=f(1)+f\left(x_{0}\right)+f\left(x_{1}\right)+\cdots+f\left(x_{r}\right) \\
& =1-1=0,
\end{aligned}
$$

so that $f$ is not invertible by Lemma 2.2. This contradiction proves that $\psi(x, k)=k$ implies $x=1$.

If we assume (b), then for any multiplicative function $f$,

$$
S_{f}(k)=\sum_{\psi(x, k)=k} f(x)=f(1)=1,
$$

so that $f$ is invertible, again by Lemma 2.2.

Lemma 2.18. Let $T, \psi$ and $\theta_{p}$ be as in Lemma 2.9. We assume that each multiplicative function is invertible with respect to $\psi$ and that (1.4) is valid. We fix a prime $p$ and write $\theta=\theta_{p}$. If $\left(p^{\lambda}, p^{\mu}\right) \in T$, then for any multiplicative function $f$,

$$
f\left(p^{\theta(\lambda, \mu)}\right)=f\left(p^{\lambda}\right) f\left(p^{\mu}\right)-f^{-1}\left(p^{\theta(\lambda, \mu)}\right)-\sum(\lambda, \mu)
$$

where

$$
\sum(\lambda, \mu)=\sum_{\substack{a, b, x, y \\(x, y) \neq(0,0) \\ x \neq \lambda, y \neq \mu \\ \theta(a, x)=\lambda, \theta(b, y)=\mu}} f\left(p^{x}\right) f\left(p^{y}\right) f^{-1}\left(p^{\theta(a, b)}\right) .
$$

Proof. Taking $m=p^{\lambda}$ and $n=p^{\mu}$ in (1.4), we obtain

$$
f\left(p^{\theta(\lambda, \mu)}\right)=\sum_{\substack{\theta(a, x)=\lambda \\ \theta(b, y)=\mu}} f\left(p^{x}\right) f\left(p^{y}\right) f^{-1}\left(p^{\theta(a, b)}\right) G\left(p^{a}, p^{b}\right) .
$$

From the definition of $G$ given in (1.3), in the above sum either $a=b=0$ or $a>0$ and $b>0$. Hence

$$
f\left(p^{\theta(\lambda, \mu)}\right)=f\left(p^{\lambda}\right) f\left(p^{\mu}\right)-\sum_{\substack{\theta(a, x)=\lambda \\ \theta(b, y)=\mu \\ a>0, b>0}} f\left(p^{x}\right) f\left(p^{y}\right) f^{-1}\left(p^{\theta(a, b)}\right) .
$$


By Lemma 2.17, $\psi(x, k)=k$ if and only if $x=1$ for each positive integer $k$. It follows that $\theta(x, y)=x$ if and only if $y=0$. Hence the conditions $a>0$ and $b>0$ in the sum on the right of $(2.20)$ can be replaced by $x \neq \lambda$ and $y \neq \mu$. In this sum the term corresponding to $(x, y)=(0,0)$ is $f^{-1}\left(p^{\theta(\lambda, \mu)}\right)$. We separate this term from this sum and we obtain (2.19). This completes the proof of Lemma 2.18.

Remark 2.21. If each multiplicative function is invertible with respect to $\psi$, as observed in the proof of Lemma 2.18 , we have

$$
\theta(x, y)=x \text { if and only if } y=0 .
$$

Let us recall also Property (e) of Lemma 2.9:

$$
\theta(x, y)=0 \text { if and only if } x=y=0 .
$$

Moreover, from Lemma $2.9(\mathrm{~g})$ and (h), the law $\lambda, \mu \mapsto \theta(\lambda, \mu)$ is commutative and associative so that we can define by induction for $k \geq 3$,

$$
\theta\left(a_{1}, a_{2}, \ldots, a_{k}\right)=\theta\left(\theta\left(a_{1}, a_{2}, \ldots, a_{k-1}\right), a_{k}\right)
$$

and for any permutation $\sigma:\{1,2, \ldots, k\} \rightarrow\{1,2, \ldots, k\}$ we have

$$
\theta\left(a_{1}, a_{2}, \ldots, a_{k}\right)=\theta\left(a_{\sigma(1)}, a_{\sigma(2)}, \ldots, a_{\sigma(k)}\right) .
$$

By Lemma 2.6, for any prime power $p^{t}, t>0$, we have

$$
\begin{aligned}
f^{-1}\left(p^{t}\right) & \left.=-\sum_{\substack{\theta(x, y)=t \\
y<t}} f\left(p^{x}\right) f^{-1}\left(p^{y}\right)\right) \\
& =-f\left(p^{t}\right)-\sum_{\substack{\theta(x, y)=t \\
0<y<t}} f\left(p^{x}\right) f^{-1}\left(p^{y}\right) .
\end{aligned}
$$

We frequently make use of $(2.19)$ to $(2.26)$ in the subsequent sections.

\section{Theorem 3.1}

In this section we prove

Theorem 3.1. Fix a prime $p$. Let $T, \psi$ and $\theta=\theta_{p}$ be as in Lemma 2.9. Assume that the identical equation (1.4) holds for all multiplicative functions $f$. Then for any non-negative integers $\alpha, \beta$ and $\gamma$,

$$
\theta(\alpha, \beta)=\theta(\alpha, \gamma) \text { implies } \beta=\gamma .
$$

Proof. First we prove that if $\alpha$ and $\beta$ are non-negative integers with $\theta(\alpha, \alpha)=\theta(\alpha, \beta)$, then $\alpha=\beta$. If $\alpha=0$ then from (2.22) and (2.23)

$$
\beta=\theta(0, \beta)=\theta(\alpha, \beta)=\theta(\alpha, \alpha)=\theta(0,0)=0=\alpha
$$

while, if $\beta=0$

$$
\alpha=\theta(\alpha, 0)=\theta(\alpha, \beta)=\theta(\alpha, \alpha)
$$


so that $\alpha=0=\beta$ from (2.22).

Suppose that $\alpha$ and $\beta$ are positive integers with $\theta(\alpha, \alpha)=\theta(\alpha, \beta)$. Let $\alpha \neq \beta$; we deduce a contradiction. We define the multiplicative function $f$ by

$$
f\left(p^{x}\right)= \begin{cases}1, & \text { if } x=0 \text { or } \alpha \\ 0, & \text { if } x \notin\{0, \alpha\} .\end{cases}
$$

Since $\alpha \neq 0$, it follows from (2.22) and (2.23) that $\theta(\alpha, \alpha) \notin\{0, \alpha\}$ so that $f\left(p^{\theta(\alpha, \alpha)}\right)=0$. Taking $\lambda=\mu=\alpha$ in (2.19) and with $f$ as in (3.2), we obtain

$$
0=f\left(p^{\theta(\alpha, \alpha)}\right)=1-f^{-1}\left(p^{\theta(\alpha, \alpha)}\right)-\sum(\alpha, \alpha),
$$

where

$$
\sum(\alpha, \alpha)=\sum_{\substack{(x, y) \neq(0,0) \\ x \neq \alpha, y \neq \alpha \\ \theta(a, x)=\alpha, \theta(b, y)=\alpha}} f\left(p^{x}\right) f\left(p^{y}\right) f^{-1}\left(p^{\theta(a, b)}\right) .
$$

From the definition of $f$ given in (3.2), in the sum defining $\sum(\alpha, \alpha)$, the non-zero terms correspond to the values of $x$ and $y$, where $x \in\{0, \alpha\}$ and $y \in\{0, \alpha\}, x \neq \alpha, y \neq \alpha$ and $(x, y) \neq(0,0)$. Since no such choice of $(x, y)$ is possible, it follows that $\sum(\alpha, \alpha)$ is zero. Hence from (3.3),

$$
f^{-1}\left(p^{\theta(\alpha, \alpha)}\right)=1 \text {. }
$$

From (3.2), $f\left(p^{\beta}\right)=0$. Again by (2.19),

$$
0=f\left(p^{\theta(\alpha, \beta)}\right)=-f^{-1}\left(p^{\theta(\alpha, \beta)}\right)-\sum(\alpha, \beta),
$$

where

$$
\sum(\alpha, \beta)=\sum_{\substack{(x, y) \neq(0,0) \\ x \neq \alpha, y \neq \beta \\ \theta(a, x)=\alpha, \theta(b, y)=\beta}} f\left(p^{x}\right) f\left(p^{y}\right) f^{-1}\left(p^{\theta(a, b)}\right) .
$$

But, from (3.2), in order that $f\left(p^{x}\right) f\left(p^{y}\right) \neq 0$ we must have $x \in\{0, \alpha\}$, $y \in\{0, \alpha\}$, and then $f\left(p^{x}\right) f\left(p^{y}\right)=1$. With the conditions $(x, y) \neq(0,0)$, $x \neq \alpha, y \neq \beta$, the only possibility is $x=0, y=\alpha$. From (2.22), the only solution in $a$ of $\theta(a, 0)=\alpha$ is $a=\alpha$. So

$$
\sum(\alpha, \beta)=\sum_{\theta(b, \alpha)=\beta} f^{-1}\left(p^{\theta(\alpha, b)}\right) .
$$

However, from (2.24) and (2.25), $\theta(b, \alpha)=\beta$ implies

$$
\begin{array}{r}
\theta(\alpha, \beta)=\theta(\alpha, \theta(b, \alpha))=\theta(\alpha, b, \alpha)=\theta(b, \alpha, \alpha) \\
=\theta(b, \theta(\alpha, \alpha))=\theta(b, \theta(\alpha, \beta)),
\end{array}
$$


so that, from (2.22), $b=0$ and hence $\alpha=\beta$. Since $\alpha \neq \beta$ is assumed it follows that the sum on the right of (3.6) is an empty sum. Thus $\sum(\alpha, \beta)=$ 0 . Using this in (3.5), we obtain

$$
f^{-1}\left(p^{\theta(\alpha, \beta)}\right)=0
$$

Since $\theta(\alpha, \alpha)=\theta(\alpha, \beta),(3.4)$ and (3.7) contradict each other. Hence $\alpha=\beta$.

We now return to the general case. Let $\alpha, \beta$ and $\gamma$ be non-negative integers with $\theta(\alpha, \beta)=\theta(\alpha, \gamma)$. We want to show that $\beta=\gamma$. If $\alpha \beta \gamma=0$, it easily follows that $\beta=\gamma$. So, we may assume that $\alpha, \beta$ and $\gamma$ are positive integers. If $\alpha=\beta$ or $\alpha=\gamma$, we obtain $\beta=\gamma$ by the previous case. Hence we can assume that $\alpha \neq \beta$ and $\alpha \neq \gamma$. Let $\beta \neq \gamma$ so that $\alpha, \beta, \gamma$ are pairwise distinct. We deduce a contradiction. Note that $\theta(\alpha, \beta) \neq 0, \alpha, \beta, \gamma$. We define the multiplicative function $f$ by

$$
f\left(p^{x}\right)= \begin{cases}1, & \text { if } x=0 \\ 0, & \text { if } x \notin\{0, \alpha, \beta, \gamma\}\end{cases}
$$

the values $f\left(p^{\alpha}\right), f\left(p^{\beta}\right)$ and $f\left(p^{\gamma}\right)$ remain to be fixed. From (2.19) with $f$ as in (3.8), we obtain

$$
0=f\left(p^{\theta(\alpha, \beta)}\right)=f\left(p^{\alpha}\right) f\left(p^{\beta}\right)-f^{-1}\left(p^{\theta(\alpha, \beta)}\right)-\sum(\alpha, \beta)
$$

where

$$
\sum(\alpha, \beta)=\sum_{\substack{(x, y) \neq(0,0) \\ x \in\{0, \beta, \gamma\} \\ y \in\{0, \alpha, \gamma\} \\ \theta(a, x)=\alpha, \theta(b, y)=\beta}} f\left(p^{x}\right) f\left(p^{y}\right) f^{-1}\left(p^{\theta(a, b)}\right) .
$$

For positive integers $m$ and $n$, let

$$
N(m, n)=\sum_{\theta(a, m)=n} 1
$$

and

$$
F(m, n)=\sum_{\theta(a, m)=n} f^{-1}\left(p^{a}\right)
$$

We have

$$
\sum(\alpha, \beta)=\sum_{\substack{x=0 \\ y=\alpha}}+\sum_{\substack{x=0 \\ y=\gamma}}+\sum_{\substack{x=\beta \\ y=0}}+\sum_{\substack{x=\beta \\ y=\alpha}}+\sum_{\substack{x=\beta \\ y=\gamma}}
$$




$$
\begin{aligned}
& +\sum_{\substack{x=\gamma \\
y=0}}+\sum_{\substack{x=\gamma \\
y=\alpha}}+\sum_{\substack{x=\gamma \\
y=\gamma}} \\
& =\sum_{i=1}^{8} \sum_{i}, \text { say }
\end{aligned}
$$

where, for instance in $\sum_{1}$, it should be understood that $x$ and $y$ are fixed and equal to 0 and $\alpha$ respectively so that

$$
\sum_{1}=\sum_{\substack{x=0 \\ y=\alpha}}=f\left(p^{0}\right) f\left(p^{\alpha}\right) \sum_{\substack{a, b \\ \theta(a, 0)=\alpha, \theta(b, \alpha)=\beta}} f^{-1}\left(p^{\theta(a, b)}\right) .
$$

Remark 3.13. If $\theta(b, \gamma)=\beta$ for some $b$, then from (2.24) and (2.25)

$$
\begin{aligned}
\theta(\alpha, \beta)=\theta(\alpha, \theta(b, \gamma))=\theta(\alpha, b, \gamma)=\theta(\alpha, \gamma, b) & \\
& =\theta(\theta(\alpha, \gamma), b)=\theta(\theta(\alpha, \beta), b),
\end{aligned}
$$

so that, from (2.22), $b=0$ and $\gamma=\beta$. Since $\gamma \neq \beta$ is assumed, it follows that $\theta(b, \gamma)=\beta$ is not solvable. Therefore the sums in (3.12) corresponding to $y=\gamma$ are empty sums, i.e. $\sum_{2}, \sum_{5}$ and $\sum_{8}$ are empty sums.

We have, since, from (2.22), $\theta(a, 0)=\alpha$ implies $a=\alpha$,

$$
\begin{aligned}
\sum_{1}=\sum_{\substack{x=0 \\
y=\alpha}}=f\left(p^{\alpha}\right) \sum_{\theta(a, 0)=\alpha, \theta(b, \alpha)=\beta} f^{-1}\left(p^{\theta(a, b)}\right) \\
=f\left(p^{\alpha}\right) \sum_{\theta(b, \alpha)=\beta} f^{-1}\left(p^{\theta(\alpha, b)}\right) \\
=f\left(p^{\alpha}\right) f^{-1}\left(p^{\beta}\right) \sum_{\theta(b, \alpha)=\beta} 1 \\
=f\left(p^{\alpha}\right) f^{-1}\left(p^{\beta}\right) N(\alpha, \beta) .
\end{aligned}
$$

Similarly,

$$
\sum_{3}=\sum_{\substack{x=\beta \\ y=0}}=f\left(p^{\beta}\right) \sum_{\theta(a, \beta)=\alpha} f^{-1}\left(p^{\theta(a, \beta)}\right)=f\left(p^{\beta}\right) f^{-1}\left(p^{\alpha}\right) N(\beta, \alpha) .
$$

If $\theta(a, \beta)=\alpha$ and $\theta(b, \alpha)=\beta$, since $\theta(x, y) \geq \max \{x, y\}$, it follows that $\beta \leq \alpha$ and $\alpha \leq \beta$ so that $\alpha=\beta$.Since $\alpha \neq \beta$ is assumed it follows that

$$
\sum_{4}=\sum_{\substack{x=\beta \\ y=\alpha}}=f\left(p^{\beta}\right) f\left(p^{\alpha}\right) \sum_{\substack{\theta(a, \beta)=\alpha \\ \theta(b, \alpha)=\beta}} f^{-1}\left(p^{\theta(a, b)}\right)=\text { Empty sum }=0 .
$$


Now,

$$
\sum_{6}=\sum_{\substack{x=\gamma \\ y=0}}=f\left(p^{\gamma}\right) \sum_{\theta(a, \gamma)=\alpha} f^{-1}\left(p^{\theta(a, \beta)}\right) .
$$

If $\theta(a, \gamma)=\alpha$ and $\theta(b, \alpha)=\beta$, from (2.24) and (2.25) we have

$$
\theta(\alpha, \beta)=\theta(\theta(a, b), \theta(\alpha, \gamma))=\theta(\theta(a, b), \theta(\alpha, \beta))
$$

so that $\theta(a, b)=0$ and hence $a=b=0$ so that $\gamma=\alpha$ and $\alpha=\beta$. Since this is not the case, we obtain

$$
\sum_{7}=\sum_{\substack{x=\gamma \\ y=\alpha}}=f\left(p^{\gamma}\right) f\left(p^{\alpha}\right) \sum_{\substack{\theta(a, \gamma)=\alpha \\ \theta(b, \alpha)=\beta}} f^{-1}\left(p^{\theta(a, b)}\right)=\text { Empty sum }=0 .
$$

From (3.9), (3.12), Remark 3.13, and (3.14)-(3.18), we obtain

$$
\begin{aligned}
& f^{-1}\left(p^{\theta(\alpha, \beta)}\right)=f\left(p^{\alpha}\right) f\left(p^{\beta}\right)-f\left(p^{\alpha}\right) f^{-1}\left(p^{\beta}\right) N(\alpha, \beta) \\
&-f\left(p^{\beta}\right) f^{-1}\left(p^{\alpha}\right) N(\beta, \alpha)-f\left(p^{\gamma}\right) \sum_{\theta(a, \gamma)=\alpha} f^{-1}\left(p^{\theta(a, \beta)}\right) .
\end{aligned}
$$

Since $\theta(\alpha, \beta)=\theta(\beta, \alpha)$, interchanging the roles of $\alpha$ and $\beta$ in (3.19) we obtain

$$
\begin{aligned}
f^{-1}\left(p^{\theta(\alpha, \beta)}\right)= & f^{-1}\left(p^{\theta(\beta, \alpha)}\right)=f\left(p^{\alpha}\right) f\left(p^{\beta}\right)-f\left(p^{\beta}\right) f^{-1}\left(p^{\alpha}\right) N(\beta, \alpha) \\
& -f\left(p^{\alpha}\right) f^{-1}\left(p^{\beta}\right) N(\alpha, \beta)-f\left(p^{\gamma}\right) \sum_{\theta(a, \gamma)=\beta} f^{-1}\left(p^{\theta(a, \alpha)}\right) .
\end{aligned}
$$

Equating (3.19) and (3.20) and cancelling the like terms we obtain

$$
f\left(p^{\gamma}\right) \sum_{\theta(a, \gamma)=\alpha} f^{-1}\left(p^{\theta(a, \beta)}\right)=f\left(p^{\gamma}\right) \sum_{\theta(a, \gamma)=\beta} f^{-1}\left(p^{\theta(a, \alpha)}\right) .
$$

By Remark 3.13, the sum on the right hand side of (3.21) is an empty sum so that its value is zero. Using this in (3.19), we get

$$
\begin{array}{r}
f^{-1}\left(p^{\theta(\alpha, \beta)}\right)=f\left(p^{\alpha}\right) f\left(p^{\beta}\right)-f\left(p^{\alpha}\right) f^{-1}\left(p^{\beta}\right) N(\alpha, \beta) \\
-f\left(p^{\beta}\right) f^{-1}\left(p^{\alpha}\right) N(\beta, \alpha)
\end{array}
$$

In a similar way we can prove that

$$
\begin{aligned}
f^{-1}\left(p^{\theta(\alpha, \gamma)}\right)=f\left(p^{\alpha}\right) f\left(p^{\gamma}\right)-f\left(p^{\alpha}\right) f^{-1}\left(p^{\gamma}\right) N(\alpha, \gamma) & \\
& -f\left(p^{\gamma}\right) f^{-1}\left(p^{\alpha}\right) N(\gamma, \alpha)
\end{aligned}
$$


We shall now find $f^{-1}\left(p^{\alpha}\right), f^{-1}\left(p^{\beta}\right)$ and $f^{-1}\left(p^{\gamma}\right)$. Observing that, by (2.22), the conditions $\theta(x, y)=\alpha$ and $0<y<\alpha$ imply $x \notin\{0, \alpha\}$ so that, from (3.8), $x \in\{\beta, \gamma\}$, we have from (2.26)

$$
\begin{aligned}
& f^{-1}\left(p^{\alpha}\right)=-f\left(p^{\alpha}\right)-\sum_{\substack{\theta(x, y)=\alpha \\
0<<y<\alpha}} f\left(p^{x}\right) f^{-1}\left(p^{y}\right) \\
& =-f\left(p^{\alpha}\right)-f\left(p^{\beta}\right) \sum_{\substack{\theta(y, \beta)=\alpha \\
0<y<\alpha}} f^{-1}\left(p^{y}\right)-f\left(p^{\gamma}\right) \sum_{\substack{\theta(y, \gamma)=\alpha \\
0<y<\alpha}} f^{-1}\left(p^{y}\right) \\
& =-f\left(p^{\alpha}\right)-f\left(p^{\beta}\right) \sum_{\substack{\theta(a, \beta)=\alpha \\
f^{\prime}}} f^{-1}\left(p^{a}\right)-f\left(p^{\gamma}\right) \sum_{\theta(b, \gamma)=\alpha} f^{-1}\left(p^{b}\right) \\
& =-f\left(p^{\alpha}\right)-f\left(p^{\beta}\right) F(\beta, \alpha)-f\left(p^{\gamma}\right) F(\gamma, \alpha) .
\end{aligned}
$$

Also

$$
f^{-1}\left(p^{\beta}\right)=-f\left(p^{\beta}\right)-\sum_{\substack{\theta(x, y)=\beta \\ 0<y<\beta}} f\left(p^{x}\right) f^{-1}\left(p^{y}\right) .
$$

From (2.22), the conditions imposed in the above sum imply that $x \notin\{0, \beta\}$. In order that the sum does not contain terms which become zero the only possible choices are $x=\gamma$ or $x=\alpha$. However, if $x=\gamma$, by Remark 3.13 the sum corresponding to this choice will be empty. Hence

$$
f^{-1}\left(p^{\beta}\right)=-f\left(p^{\beta}\right)-f\left(p^{\alpha}\right) F(\alpha, \beta) .
$$

Similarly,

$$
f^{-1}\left(p^{\gamma}\right)=-f\left(p^{\gamma}\right)-f\left(p^{\alpha}\right) F(\alpha, \gamma) .
$$

Making use of (3.24)-(3.26) in (3.22) and (3.23) and equating the two results we obtain

$$
\begin{gathered}
f\left(p^{\alpha}\right) f\left(p^{\beta}\right)\{1+N(\alpha, \beta)+N(\beta, \alpha)\} \\
+f^{2}\left(p^{\alpha}\right) N(\alpha, \beta) F(\alpha, \beta)+f^{2}\left(p^{\beta}\right) N(\beta, \alpha) F(\beta, \alpha) \\
+f\left(p^{\beta}\right) f\left(p^{\gamma}\right) N(\beta, \alpha) F(\gamma, \alpha) \\
=f\left(p^{\alpha}\right) f\left(p^{\gamma}\right)\{1+N(\alpha, \gamma)+N(\gamma, \alpha)\} \\
+f^{2}\left(p^{\alpha}\right) N(\alpha, \gamma) F(\alpha, \gamma)+f^{2}\left(p^{\gamma}\right) N(\gamma, \alpha) F(\gamma, \alpha) \\
+f\left(p^{\gamma}\right) f\left(p^{\beta}\right) N(\gamma, \alpha) F(\beta, \alpha) .
\end{gathered}
$$

We now take $f\left(p^{\gamma}\right)=0$ and $f\left(p^{\alpha}\right)=1$ in (3.27). We get after transposing the terms,

$$
\begin{aligned}
0= & f\left(p^{\beta}\right)\{1+N(\alpha, \beta)+N(\beta, \alpha)\} \\
& +N(\alpha, \beta) F(\alpha, \beta)+f^{2}\left(p^{\beta}\right) N(\beta, \alpha) F(\beta, \alpha)-N(\alpha, \gamma) F(\alpha, \gamma) .
\end{aligned}
$$

To obtain a contradiction from (3.28) we require the following: 
Lemma 3.29. Let $\lambda, \mu$ be two distinct positive integers and $\varphi$ a multiplicative function defined by

$$
\varphi\left(p^{x}\right)= \begin{cases}1, & \text { if } x=0 \\ 0, & \text { if } x \notin\{0, \lambda, \mu\}\end{cases}
$$

$\varphi\left(p^{\lambda}\right)$ and $\varphi\left(p^{\mu}\right)$ being kept arbitrary. We set $\varphi\left(p^{\lambda}\right)=\rho$. Then, if we define similarly as in (3.11) $F(\lambda, \mu)=\sum_{\theta(a, \lambda)=\mu} \varphi^{-1}\left(p^{a}\right)$, we have $F(\lambda, \mu)=0$ or $\pm \rho^{r}$ for some positive integer $r$.

Proof. If $F(\lambda, \mu)$ is an empty sum its value is zero. We may assume that it is a non empty sum. In this case we show that $F(\lambda, \mu)= \pm \rho^{r}$. We have by $(2.26)$

$$
\begin{aligned}
F(\lambda, \mu) & =\sum_{\theta(a, \lambda)=\mu} \varphi^{-1}\left(p^{a}\right) \\
& =\sum_{\theta(a, \lambda)=\mu}\left\{-\varphi\left(p^{a}\right)-\sum_{\substack{\theta\left(a_{1}, b_{1}\right)=a \\
0<b_{1}<a}} \varphi\left(p^{a_{1}}\right) \varphi^{-1}\left(p^{b_{1}}\right)\right\} \\
& =-\sum_{\theta(a, \lambda)=\mu} \varphi\left(p^{a}\right)-\sum_{\theta(a, \lambda)=\mu} \sum_{\substack{\theta\left(a_{1}, b_{1}\right)=a \\
0<b_{1}<a}} \varphi\left(p^{a_{1}}\right) \varphi^{-1}\left(p^{b_{1}}\right) .
\end{aligned}
$$

In the inner most sum on the right hand side of (3.30), $0<a_{1}<a<\mu$. Hence $a_{1} \neq \mu$. From the definition of the function $\varphi$, the only possible choice of $a_{1}$ in order that this sum contains a non-zero term is $a_{1}=\lambda$, so that

$$
\begin{aligned}
F(\lambda, \mu) & =-\sum_{\theta(a, \lambda)=\mu} \varphi\left(p^{a}\right)-\sum_{\theta(a, \lambda)=\mu} \sum_{\substack{\theta\left(\lambda, b_{1}\right)=a \\
0<b_{1}<a}} \rho \varphi^{-1}\left(p^{b_{1}}\right) \\
& =-K_{1}-K_{2}, \text { say. }
\end{aligned}
$$

Clearly,

$$
K_{1}= \begin{cases}\rho, & \text { if } \mu=\theta(\lambda, \lambda) \\ 0, & \text { otherwise. }\end{cases}
$$

We note that $K_{1} \neq 0$ implies that the sum defining $K_{2}$ is an empty sum so that $K_{2}=0$. For, let $K_{1}=\rho$ so that $\mu=\theta(\lambda, \lambda)$. If $K_{2}$ is not an empty sum, we would have $a=\theta\left(\lambda, b_{1}\right), \mu=\theta(a, \lambda), 0<b_{1}<a$, so that

$$
\mu=\theta(a, \lambda)=\theta\left(\theta\left(\lambda, b_{1}\right), \lambda\right)=\theta\left(b_{1}, \theta(\lambda, \lambda)\right)=\theta\left(b_{1}, \mu\right)
$$

and hence $b_{1}=0$. But $b_{1}>0$. Therefore if $K_{1} \neq 0$ then $K_{2}=0$ and hence $F(\lambda, \mu)=-K_{1}-K_{2}=-\rho$. So let $K_{1}=0$ so that $F(\lambda, \mu)=-K_{2}$. Again 
by $(2.26)$,

$$
\begin{aligned}
& K_{2}=\sum_{\theta(a, \lambda)=\mu} \sum_{\substack{\theta\left(\lambda, b_{1}\right)=a \\
0<b_{1}<a}} \rho\left\{-\varphi\left(p^{b_{1}}\right)-\sum_{\substack{\theta\left(a_{2}, b_{2}\right)=b_{1} \\
0<b_{2}<b_{1}}} \varphi\left(p^{a_{2}}\right) \varphi^{-1}\left(p^{b_{2}}\right)\right\} \\
& =-\sum_{\theta(a, \lambda)=\mu} \sum_{\substack{\theta\left(\lambda, b_{1}\right)=a \\
0<b_{1}<a}} \rho \varphi\left(p^{b_{1}}\right)-\sum_{\theta(a, \lambda)=\mu} \sum_{\substack{\theta\left(\lambda, b_{1}\right)=a \\
0<b_{1}<a}} \rho \\
& \times \sum_{\substack{\theta\left(a_{2}, b_{2}\right)=b_{1} \\
0<b_{2}<b_{1}}} \varphi\left(p^{a_{2}}\right) \varphi^{-1}\left(p^{b_{2}}\right) .
\end{aligned}
$$

As before we can assume that $a_{2}=\lambda$ in the inner most sum on the right hand side above. So, we get

$$
K_{2}=-K_{3}-K_{4},
$$

where

$$
K_{4}=\rho^{2} \sum_{\theta(a, \lambda)=\mu} \sum_{\substack{\theta\left(\lambda, b_{1}\right)=a \\ 0<b_{1}<a}} \sum_{\substack{\theta\left(\lambda, b_{2}\right)=b_{1} \\ 0<b_{2}<b_{1}}} \varphi^{-1}\left(p^{b_{2}}\right)
$$

and

$$
K_{3}= \begin{cases}\rho^{2}, & \text { if } \mu=\theta(\theta(\lambda, \lambda), \lambda) \\ 0, & \text { otherwise. }\end{cases}
$$

We write $\lambda_{2}=\theta(\lambda, \lambda), \lambda_{3}=\theta\left(\lambda, \lambda_{2}\right), \lambda_{4}=\theta\left(\lambda, \lambda_{3}\right)$ and so on.

As before we note that $K_{3} \neq 0$ implies $K_{4}$ is an empty sum so that

$$
F(\lambda, \mu)=-K_{2}=-\left(-K_{3}-K_{4}\right)=K_{3}+K_{4}=\rho^{2} .
$$

If $K_{3}=0, F(\lambda, \mu)=K_{4}$. For a given $a$ with $\theta(a, \lambda)=\mu$, this procedure can not be continued indefinitely since we obtain a strictly decreasing sequence of positive integers with $\mu>a>b_{1}>b_{2}>\cdots>0$. Hence for some positive integer $r(a)$ we get that $\varphi^{-1}\left(p^{b_{r(a)}}\right)=-\varphi\left(p^{b_{r(a)}}\right)$ and

$$
\begin{aligned}
F(\lambda, \mu) & = \pm \sum_{\theta(a, \lambda)=\mu} \sum_{\substack{\theta\left(\lambda, b_{1}\right)=a \\
0<b_{1}<a}} \cdots \sum_{\begin{array}{c}
\theta\left(\lambda, b_{r}(a)\right)=b_{r(a)-1} \\
\left.0<b_{r(a)}\right) b_{r(a)-1}
\end{array}} \rho^{r(a)} \\
& = \pm \sum_{\theta(a, \lambda)=\mu} \sum_{\begin{array}{c}
\theta\left(\lambda, b_{1}\right)=a \\
0<b_{1}<a \\
b_{1}=\lambda_{r(a)+2} \\
r(a)
\end{array}} \rho^{r(a)} . \\
& = \pm \sum_{\substack{\theta(a, \lambda)=\mu \\
a=\lambda_{r(a)+3}}}
\end{aligned}
$$


Since $\theta(\lambda, \lambda)=\theta(\lambda, \mu)$ implies $\lambda=\mu$ (which has been proved earlier), $\lambda_{k}=$ $\lambda_{\ell}$ implies $k=\ell$. Hence there can be at most one term in the above sum so that $F(\lambda, \mu)= \pm \rho^{r(a)}$, which completes the proof of Lemma 3.29.

We now return to the equation (3.28). We apply Lemma 3.29 with $\lambda=\beta$, $\mu=\alpha, \varphi=f$ (on noting that we have chosen $f\left(p^{\gamma}\right)=0$ in (3.8)) so that $F(\beta, \alpha)=0$ or $\pm\left(f\left(p^{\beta}\right)\right)^{r}$ for some positive integer $r$. If $F(\beta, \alpha)=0,(3.28)$ reduces to

$$
\begin{aligned}
0=f\left(p^{\beta}\right)\{1+N(\alpha, \beta)+ & N(\beta, \alpha)\} \\
& +N(\alpha, \beta) F(\alpha, \beta)-N(\alpha, \gamma) F(\alpha, \gamma) .
\end{aligned}
$$

Since the coefficient of $f\left(p^{\beta}\right)$ is positive in the above equation, we can easily assign a value to $f\left(p^{\beta}\right)$ which does not satisfy the equation (3.31). If $F(\beta, \alpha)= \pm\left(f\left(p^{\beta}\right)^{r}\right.$, we obtain from (3.28)

$$
\begin{aligned}
0= \pm N(\beta, \alpha)\left(f\left(p^{\beta}\right)^{r+2}+\right. & f\left(p^{\beta}\right)\{1+N(\alpha, \beta)+N(\beta, \alpha)\} \\
& -N(\alpha, \gamma) F(\alpha, \gamma)+N(\alpha, \beta) F(\alpha, \beta) .
\end{aligned}
$$

Since $F(\beta, \alpha) \neq 0$ implies $N(\beta, \alpha)>0$, we can regard equation (3.32) as a polynomial equation of degree $r+2$ in the variable $f\left(p^{\beta}\right)$. If $f\left(p^{\beta}\right)$ is chosen in such a way that $f\left(p^{\beta}\right)$ equals none of the $r+2$ roots of the equation (3.32), we obtain a contradiction. This completes the proof of Theorem 3.1.

\section{Theorem 4.1}

In this section we prove

Theorem 4.1. (With the hypothesis of Theorem 3.1). If $\alpha, \beta, \gamma$ and $\delta$ are non-negative integers such that

$$
\theta(\alpha, \beta)=\theta(\gamma, \delta),
$$

then either $\alpha=\theta(\gamma, c)$ for some $c \geq 0$ or $\beta=\theta(\delta, d)$ for some $d \geq 0$.

Proof. Most of the arguments we shall use in this proof were already used in the proof of Theorem 3.1, so that we shall omit some details.

We first prove that if $\alpha$ and $\beta$ are non-negative integers, then

$$
\theta(\alpha, \alpha)=\theta(\beta, \beta) \quad \text { implies } \alpha=\beta \text {. }
$$

We may assume that $\alpha$ and $\beta$ are positive integers. Let $\alpha \neq \beta$. We deduce a contradiction. Define the multiplicative function $f$ by

$$
f\left(p^{x}\right)= \begin{cases}1, & \text { if } x=0 \text { or } \beta \\ 0, & \text { otherwise. }\end{cases}
$$


First, we observe from (2.22) and (2.23) that $\theta(\alpha, \alpha)=\theta(\beta, \beta) \notin\{0, \beta\}$ so that $f\left(p^{\theta(\alpha, \alpha)}\right)=0$. Taking $\lambda=\mu=\alpha$ in (2.19) and $f$ as in (4.2), we obtain

$$
0=f\left(p^{\theta(\alpha, \alpha)}\right)=-f^{-1}\left(p^{\theta(\alpha, \alpha)}\right)-\sum(\alpha, \alpha)
$$

where

$$
\sum(\alpha, \alpha)=\sum_{\substack{(x, y) \neq(0,0) \\ x \in\{0, \beta\}, y \in\{0, \beta\} \\ \theta(a, x)=\alpha, \theta(b, y)=\alpha}} f\left(p^{x}\right) f\left(p^{y}\right) f^{-1}\left(p^{\theta(a, b)}\right) .
$$

We have, as in (3.12)

$$
\sum(\alpha, \alpha)=\sum_{\substack{x=0 \\ y=\beta}}+\sum_{\substack{x=\beta \\ y=0}}+\sum_{\substack{x=\beta \\ y=\beta}}
$$

If $\theta(c, \beta)=\alpha$ for some $c$, we have from (2.23) and (2.24)

$$
\theta(\alpha, \alpha)=\theta(c, \beta, c, \beta)=\theta(\theta(c, c), \theta(\beta, \beta))=\theta(\theta(c, c), \theta(\alpha, \alpha)),
$$

so that $\theta(c, c)=0$ and hence $c=0$ so that $\alpha=\beta$. Since $\alpha \neq \beta$ is assumed, it follows that $\theta(c, \beta)=\alpha$ has no solutions. Hence each sum on the right hand side of (4.4) is empty so that $\sum(\alpha, \alpha)=0$. Using this in (4.3), we obtain

$$
f^{-1}\left(p^{\theta(\alpha, \alpha)}\right)=0 .
$$

We shall now evaluate $f^{-1}\left(p^{\theta(\alpha, \alpha)}\right)$ directly from (2.26). We obtain

$$
f^{-1}\left(p^{\theta(\alpha, \alpha)}\right)=-f\left(p^{\theta(\alpha, \alpha)}\right)-\sum_{\substack{\theta(x, y)=\theta(\alpha, \alpha)=\theta(\beta, \beta) \\ 0<y<\theta(\alpha, \alpha)}} f\left(p^{x}\right) f^{-1}\left(p^{y}\right) .
$$

In the above sum, from (4.2), the non-zero terms correspond to $x=0$ or $x=\beta$; but, from (2.22), $x=0$ and $\theta(x, y)=\theta(\alpha, \alpha)$ implies $y=\theta(\alpha, \alpha)$ which is forbidden, so that $x=\beta$. By Theorem 3.1, $x=\beta, \theta(x, y)=$ $\theta(\alpha, \alpha)=\theta(\beta, \beta)$ implies $y=\beta$. Hence

$$
\begin{aligned}
f^{-1}\left(p^{\theta(\alpha, \alpha)}\right) & =-f\left(p^{\theta(\alpha, \alpha)}\right)-f\left(p^{\beta}\right) f^{-1}\left(p^{\beta}\right) \\
& =0-f\left(p^{\beta}\right) f^{-1}\left(p^{\beta}\right) \\
& =-f\left(p^{\beta}\right) f^{-1}\left(p^{\beta}\right)=-f^{-1}\left(p^{\beta}\right) .
\end{aligned}
$$

Again by (2.26),

$$
f^{-1}\left(p^{\beta}\right)=-f\left(p^{\beta}\right)-\sum_{\substack{\theta(x, y)=\beta \\ 0<y<\beta}} f\left(p^{x}\right) f^{-1}\left(p^{y}\right)=-f\left(p^{\beta}\right)=-1,
$$


since, in the above sum, the non-zero terms correspond to $x \in\{0, \beta\}$ which are forbidden. Hence from (4.7),

$$
f^{-1}\left(p^{\theta(\alpha, \alpha)}\right)=1
$$

(4.5) and (4.8) contradict each other. This, proves (4.1).

Suppose now that $\alpha, \beta, \gamma$ and $\delta$ are non-negative integers such that $\theta(\alpha, \beta)=\theta(\gamma, \delta)$. We prove that

$$
\alpha=\theta(\gamma, c) \text { for some } c \geq 0 \text { or } \beta=\theta(\delta, d) \text { for some } d \geq 0 .
$$

(4.9) easily follows if $\alpha \beta \gamma \delta=0$. So we may assume that $\alpha, \beta, \gamma$ and $\delta$ are positive integers.

Let $\alpha=\beta$. If $\gamma=\delta$, then we have $\theta(\alpha, \alpha)=\theta(\gamma, \gamma)$ so that by (4.1), $\alpha=\gamma$. Hence $\alpha=\theta(\gamma, 0)$. Let $\gamma \neq \delta$. We now have

$$
\theta(\alpha, \alpha)=\theta(\gamma, \delta) \text {. }
$$

In this case we must prove (4.9) with $\beta=\alpha$. First, we observe that, if $\delta$ can be written $\delta=\theta\left(\alpha, d^{\prime}\right)$, then from (2.24) and (2.25),

$$
\theta(\alpha, \alpha)=\theta(\gamma, \delta)=\theta\left(\gamma, \theta\left(\alpha, d^{\prime}\right)\right)=\theta\left(\gamma, \alpha, d^{\prime}\right)=\theta\left(\alpha, \theta\left(\gamma, d^{\prime}\right)\right)
$$

which implies by Theorem 3.1, $\alpha=\theta\left(\gamma, d^{\prime}\right)$ so that (4.9) is satisfied. The same conclusion holds, if we assume that $\gamma=\theta\left(\alpha, c^{\prime}\right)$. Thus, if (4.9) does not hold, none of the equations

$$
\alpha=\theta(\gamma, c) ; \quad \beta=\alpha=\theta(\delta, d) ; \quad \gamma=\theta\left(\alpha, c^{\prime}\right) ; \quad \delta=\theta\left(\alpha, d^{\prime}\right)
$$

is solvable. We now define the multiplicative function $f$ by

$$
f\left(p^{x}\right)= \begin{cases}1, & \text { if } x=0 \\ 0, & \text { if } x \notin\{0, \alpha, \gamma, \delta\}\end{cases}
$$

the values $f\left(p^{\alpha}\right), f\left(p^{\gamma}\right)$ and $f\left(p^{\delta}\right)$ are left to be chosen. Taking $\lambda=\mu=\alpha$ in (2.19) with $f$ as in (4.11), we obtain

$$
0=f\left(p^{\theta(\alpha, \alpha)}\right)=f^{2}\left(p^{\alpha}\right)-f^{-1}\left(p^{\theta(\alpha, \alpha)}\right)-\sum(\alpha, \alpha)
$$

where

$$
\sum(\alpha, \alpha)=\sum_{\substack{(x, y) \neq(0,0) \\ x \in\{0, \gamma, \delta\}, y \in\{0, \gamma, \delta\} \\ \theta(a, x)=\alpha, \theta(b, y)=\alpha}} f\left(p^{x}\right) f\left(p^{y}\right) f^{-1}\left(p^{\theta(a, b)}\right) .
$$

By our assumption on the solvability of the equations mentioned above, in the sum on the right hand side of $\sum(\alpha, \alpha), x$ or $y$ can not be either $\gamma$ or $\delta$. The allowable choice is $(x, y)=(0,0)$ which is again not possible since $(x, y) \neq(0,0)$. Hence $\sum(\alpha, \alpha)=0$. Using this in (4.12) we obtain

$$
f^{-1}\left(p^{\theta(\alpha, \alpha)}\right)=f^{2}\left(p^{\alpha}\right) .
$$


By (2.26), (4.11) and by Theorem 3.1,

$$
\begin{aligned}
f^{-1}\left(p^{\theta(\alpha, \alpha)}\right) & =-f\left(p^{\theta(\alpha, \alpha)}\right)-\sum_{\substack{\theta(x, y)=\theta(\alpha, \alpha) \\
0<y<\theta(\alpha, \alpha) \\
x \in\{0, \alpha, \gamma, \delta\}}} f\left(p^{x}\right) f^{-1}\left(p^{y}\right) \\
& =-\sum_{\substack{\theta(x, y)=\theta(\alpha, \alpha)(=\theta(\gamma, \delta)) \\
0<y<\theta(\alpha, \alpha) \\
x \in\{\alpha, \gamma, \delta\}}} f\left(p^{x}\right) f^{-1}\left(p^{y}\right) .
\end{aligned}
$$

In the above sum, if $x$ is equal to $\alpha, \gamma, \delta$ respectively, then from Theorem 3.1, $y$ is equal to $\alpha, \delta, \gamma$. Further, these three values of $y$ satisfy $0<y<\theta(\alpha, \alpha)$. For instance, $\gamma \leq \theta(\gamma, \delta)=\theta(\alpha, \alpha)$ and $\gamma=\theta(\alpha, \alpha)$ is forbidden since $\gamma=\theta(\alpha, c)$ is not solvable. Therefore

$$
f^{-1}\left(p^{\theta(\alpha, \alpha)}\right)=-f\left(p^{\alpha}\right) f^{-1}\left(p^{\alpha}\right)-f\left(p^{\gamma}\right) f^{-1}\left(p^{\delta}\right)-f\left(p^{\delta}\right) f^{-1}\left(p^{\gamma}\right)
$$

By (2.26) and by our assumption,

$$
f^{-1}\left(p^{\alpha}\right)=-f\left(p^{\alpha}\right)-\sum_{\substack{\theta(x, y)=\alpha \\ 0<y<\alpha \\ x \in\{\gamma, \delta\}}} f\left(p^{x}\right) f^{-1}\left(p^{y}\right)=-f\left(p^{\alpha}\right)
$$

$$
\begin{aligned}
f^{-1}\left(p^{\gamma}\right)= & -f\left(p^{\gamma}\right)-\sum_{\substack{\theta(x, y)=\gamma \\
0<y<\gamma \\
x \in\{\alpha, \delta\}}} f\left(p^{x}\right) f^{-1}\left(p^{y}\right) \\
& =-f\left(p^{\gamma}\right)-f\left(p^{\delta}\right) \sum_{\substack{\theta(\delta, y)=\gamma \\
0<y<\gamma}} f^{-1}\left(p^{y}\right) \\
& =-f\left(p^{\gamma}\right)-f\left(p^{\delta}\right) F(\delta, \gamma),
\end{aligned}
$$

since $\gamma=\theta(\delta, y) \geq y$ implies $y \leq \gamma$, and with (2.22), $y \neq \gamma$. Similarly,

$$
f^{-1}\left(p^{\delta}\right)=-f\left(p^{\delta}\right)-\sum_{\substack{\theta(x, y)=\delta \\ 0<y<\delta \\ x \in\{\alpha, \gamma\}}} f\left(p^{x}\right) f^{-1}\left(p^{y}\right)=-f\left(p^{\delta}\right)-f\left(p^{\gamma}\right) F(\gamma, \delta) .
$$

Making use of (4.15)-(4.17) in (4.14), we obtain after simplification, (4.18) $f^{-1}\left(p^{\theta(\alpha, \alpha)}\right)=f^{2}\left(p^{\alpha}\right)+2 f\left(p^{\gamma}\right) f\left(p^{\delta}\right)+f^{2}\left(p^{\gamma}\right) F(\gamma, \delta)+f^{2}\left(p^{\delta}\right) F(\delta, \gamma)$.

Comparing (4.13) and (4.18), we obtain

$$
0=2 f\left(p^{\gamma}\right) f\left(p^{\delta}\right)+f^{2}\left(p^{\gamma}\right) F(\gamma, \delta)+f^{2}\left(p^{\delta}\right) F(\delta, \gamma) .
$$

We choose $f\left(p^{\alpha}\right)=0$. Not both the sums $F(\gamma, \delta)$ and $F(\delta, \gamma)$ can be nonempty. If $F(\gamma, \delta)$ is non-empty, so that $F(\delta, \gamma)=0$, we choose $f\left(p^{\gamma}\right)=1$. 
As in Lemma 3.29, $F(\gamma, \delta)=0$ or \pm 1 . In this case, (4.19) reduces to $0=2 f\left(p^{\delta}\right)+F(\gamma, \delta)$, so that by choosing $f\left(p^{\delta}\right) \neq-\frac{1}{2} F(\gamma, \delta)$, we obtain a contradiction. If $F(\delta, \gamma)$ is a non-empty sum (so that $F(\gamma, \delta)=0$ ) a similar contradiction can be obtained. If both $F(\gamma, \delta)$ and $F(\delta, \gamma)$ are empty sums, simply we choose $f\left(p^{\gamma}\right) f\left(p^{\delta}\right) \neq 0$ to obtain a contradiction. Thus the equation (4.19) results in a contradiction in all cases. Thus (4.9) is true when $\beta=\alpha$ and $\gamma \neq \delta$.

We now complete the remaining cases. We can assume now that $\theta(\alpha, \beta)$ $=\theta(\gamma, \delta)$, where $\alpha \neq \beta$. If $\gamma=\delta$, we obtain $\theta(\gamma, \gamma)=\theta(\alpha, \beta)$. Hence by the previous considerations, we have $\gamma=\theta(\alpha, c)$ or $\gamma=\theta(\beta, c)$. So we have $\alpha=\theta\left(\gamma, c^{\prime}\right)$ or $\beta=\theta\left(\gamma, d^{\prime}\right)=\theta\left(\delta, d^{\prime}\right)$, by Theorem 3.1. So we may assume that $\alpha \neq \beta$ and $\gamma \neq \delta$. If $\alpha=\gamma$, then $\alpha=\theta(\gamma, 0)$. If $\alpha=\delta$ then (by Theorem 3.1) $\beta=\gamma$. In this case we must show that $\alpha=\theta(\beta, c)$ or $\beta=\theta(\alpha, d)$. If these two possibilities do not occur, we may take the multiplicative function $f$ defined by

$$
f\left(p^{x}\right)= \begin{cases}1, & \text { if } x \in\{0, \alpha, \beta\} \\ 0, & \text { otherwise. }\end{cases}
$$

By (2.19) with this $f$, we can show that $f^{-1}\left(p^{\theta(\alpha, \beta)}\right)=1$ and making use of $(2.26)$ we obtain that $f^{-1}\left(p^{\theta(\alpha, \beta)}\right)=2$ leading to an absurdity.

Finally, we can now assume that $\alpha, \beta, \gamma, \delta$ are pairwise distinct positive integers with $\theta(\alpha, \beta)=\theta(\gamma, \delta)$. Suppose that the conclusion of Theorem 4.1 does not hold; this together with Theorem 3.1 implies that none of the four equations

$$
\alpha=\theta\left(\gamma, c_{1}\right) ; \quad \beta=\theta\left(\delta, c_{2}\right) ; \quad \gamma=\theta\left(\alpha, c_{3}\right) ; \quad \delta=\theta\left(\beta, c_{4}\right)
$$

is solvable.

Let $f$ be the multiplicative function defined by

$$
f\left(p^{x}\right)= \begin{cases}1, & \text { if } x=0 \\ 0, & \text { if } x \notin\{0, \gamma, \delta\}\end{cases}
$$

the values of $f\left(p^{\gamma}\right)$ and $f\left(p^{\delta}\right)$ remain to be fixed. Since $f\left(p^{\alpha}\right)=f\left(p^{\beta}\right)=0$, from (2.19), with $f$ as in (4.21) we obtain

$$
0=-f^{-1}\left(p^{\theta(\alpha, \beta)}\right)-\sum(\alpha, \beta)
$$

where

$$
\sum(\alpha, \beta)=\sum_{\substack{(x, y) \neq(0,0) \\ x \in\{0, \gamma, \delta\}, y \in\{0, \gamma, \delta\} \\ \theta(a, x)=\alpha, \theta(b, y)=\beta}} f\left(p^{x}\right) f\left(p^{y}\right) f^{-1}\left(p^{\theta(a, b)}\right) .
$$


In the sum on the right hand side of $\sum(\alpha, \beta)$, by the assumption made on the solvability of equations in (4.20), the choices $x=\gamma$ or $y=\delta$ are not permissible. Hence

$$
\sum(\alpha, \beta)=\sum_{\substack{(x, y) \neq(0,0) \\ x \in\{0,0\}, y \in\{0, \gamma\} \\ \theta(a, x)=\alpha, \theta(b, y)=\beta}} f\left(p^{x}\right) f\left(p^{y}\right) f^{-1}\left(p^{\theta(a, b)}\right),
$$

so that, as in (3.12)

$$
\sum(\alpha, \beta)=\sum_{\substack{x=0 \\ y=\gamma}}+\sum_{\substack{x=\delta \\ y=0}}+\sum_{\substack{x=\delta \\ y=\gamma}} .
$$

Remark 4.24. We may note here that $\theta(\alpha, \beta)=\theta(\gamma, \delta)$ and Theorem 3.1 imply that $\theta(b, \gamma)=\beta$ if and only if $\theta(b, \alpha)=\delta$. In particular $N(\gamma, \beta)=$ $N(\alpha, \delta)$ and $F(\gamma, \beta)=F(\alpha, \delta)$. Similarly $\theta(a, \beta)=\gamma$ if and only if $\theta(a, \delta)=$ $\alpha$. Thus $N(\beta, \gamma)=N(\delta, \alpha)$ and $F(\beta, \gamma)=F(\delta, \alpha)$.

Now by Remark 4.24 ,

$$
\begin{aligned}
\sum_{\substack{x=0 \\
y=\gamma}}=f\left(p^{\gamma}\right) \sum_{\theta(b, \gamma)=\beta} f^{-1}\left(p^{\theta(b, \alpha)}\right) & =f\left(p^{\gamma}\right) f^{-1}\left(p^{\delta}\right) N(\gamma, \beta) \\
& =f\left(p^{\gamma}\right) f^{-1}\left(p^{\delta}\right) N(\alpha, \delta)
\end{aligned}
$$

and

$$
\sum_{\substack{x=\delta \\ y=0}}=f\left(p^{\delta}\right) \sum_{\theta(a, \delta)=\alpha} f^{-1}\left(p^{\theta(a, \beta)}\right)=f\left(p^{\delta}\right) f^{-1}\left(p^{\gamma}\right) N(\delta, \alpha) .
$$

If $\theta(a, \delta)=\alpha$ and $\theta(b, \gamma)=\beta$, then by Remark 4.24, $\gamma=\theta(a, \beta), \delta=\theta(b, \alpha)$ and

$$
\theta(\alpha, \beta)=\theta(\theta(a, b), \theta(\gamma, \delta))=\theta(\theta(a, b), \theta(\theta(a, b), \theta(\alpha, \beta))),
$$

so that $\theta(a, b)=0$ and hence $a=b=0$, so that $\delta=\alpha$ and $\gamma=\beta$. This is not possible since $\alpha, \beta, \gamma$ and $\delta$ are pairwise distinct. It follows that

$$
\sum_{\substack{x=\delta \\ y=\gamma}}=\text { Empty sum }=0 .
$$

Making use of (4.25)-(4.27) in (4.23) and replacing the resulting value of $\sum(\alpha, \beta)$ in (4.22) we obtain

$$
f^{-1}\left(p^{\theta(\alpha, \beta)}\right)=-f\left(p^{\gamma}\right) f^{-1}\left(p^{\delta}\right) N(\alpha, \delta)-f\left(p^{\delta}\right) f^{-1}\left(p^{\gamma}\right) N(\delta, \alpha) .
$$

From (2.26), we can show that

$$
f^{-1}\left(p^{\gamma}\right)=-f\left(p^{\gamma}\right)-f\left(p^{\delta}\right) F(\delta, \gamma)
$$


and

$$
f^{-1}\left(p^{\delta}\right)=-f\left(p^{\delta}\right)-f\left(p^{\gamma}\right) F(\gamma, \delta) .
$$

Making use of (4.29) and (4.30) in (4.28), after simplification we obtain

$$
\begin{aligned}
f^{-1}\left(p^{\theta(\alpha, \beta)}\right)= & f\left(p^{\gamma}\right) f\left(p^{\delta}\right) N(\alpha, \delta)+f^{2}\left(p^{\gamma}\right) N(\alpha, \delta) F(\gamma, \delta) \\
& +f\left(p^{\gamma}\right) f\left(p^{\delta}\right) N(\delta, \alpha)+f^{2}\left(p^{\delta}\right) N(\delta, \alpha) F(\delta, \gamma) .
\end{aligned}
$$

Now, by (2.26), by Theorem 3.1 and since $f\left(p^{\alpha}\right)=f\left(p^{\beta}\right)=0$, we obtain

$$
\begin{aligned}
f^{-1}\left(p^{\theta(\alpha, \beta)}\right) & =-f\left(p^{\theta(\alpha, \beta)}\right)-\sum_{\substack{\theta(x, y)=\theta(\alpha, \beta)(=\theta(\gamma, \delta)) \\
0<y<\theta(\alpha, \beta) \\
x \in\{\gamma, \delta\}}} f\left(p^{x}\right) f^{-1}\left(p^{y}\right) \\
& =-f\left(p^{\gamma}\right) f^{-1}\left(p^{\delta}\right)-f\left(p^{\delta}\right) f^{-1}\left(p^{\gamma}\right) .
\end{aligned}
$$

Making use of (4.29) and (4.30) in (4.32) we obtain after simplification

$$
f^{-1}\left(p^{\theta(\alpha, \beta)}\right)=2 f\left(p^{\gamma}\right) f\left(p^{\delta}\right)+f^{2}\left(p^{\gamma}\right) F(\gamma, \delta)+f^{2}\left(p^{\delta}\right) F(\delta, \gamma)
$$

Equating (4.31) and (4.33) and transposing the terms, we get

$$
\begin{aligned}
& \quad 0=f\left(p^{\gamma}\right) f\left(p^{\delta}\right)\{2-N(\alpha, \delta)-N(\delta, \alpha)\} \\
& +f^{2}\left(p^{\gamma}\right) F(\gamma, \delta)\{1-N(\alpha, \delta)\}+f^{2}\left(p^{\delta}\right) F(\delta, \gamma)\{1-N(\delta, \alpha)\} .
\end{aligned}
$$

By Theorem 3.1, it is clear that the $N$-functions take the value 0 or 1 . Also, $N(\alpha, \delta)$ and $N(\delta, \alpha)$ can not be simultaneously unity. It follows that the coefficient of $f\left(p^{\gamma}\right) f\left(p^{\delta}\right)$ is positive. If the sum $F(\gamma, \delta)$ is non-empty, so that $F(\delta, \gamma)=0$, we choose $f\left(p^{\gamma}\right)=1$. Applying Lemma 3.29 with $\lambda=\gamma$, $\mu=\delta, \varphi=f$ (on noting that $f\left(p^{\alpha}\right)=f\left(p^{\beta}\right)=0$ ) yields $F(\gamma, \delta)=0, \pm 1$. In this case (4.34) reduces to

$$
0=f\left(p^{\delta}\right)\{2-N(\alpha, \delta)-N(\delta, \alpha)\}+F(\gamma, \delta)\{1-N(\alpha, \delta)\} .
$$

It is easy to choose $f\left(p^{\delta}\right)$ not satisfying (4.35). A similar contradiction can be obtained if $F(\delta, \gamma)$ is non-empty. If $F(\gamma, \delta)$ and $F(\delta, \gamma)$ are both empty sums, (4.34) reduces to $0=f\left(p^{\gamma}\right) f\left(p^{\delta}\right)$. This obviously gives a contradiction if $f\left(p^{\gamma}\right) f\left(p^{\delta}\right) \neq 0$. This completes the proof of Theorem 4.1.

\section{Final results}

From Lemma 2.17, Theorems 3.1 and 4.1 and the definition given in (2.13), we obtain

Theorem 5.1. If $\psi$ preserves multiplicativity, $\psi(x, y) \geq \max \{x, y\}$ for all $(x, y) \in T$, each multiplicative function is invertible with respect to $\psi$ and the identical equation (1.4) holds for all multiplicative functions, then $\psi$ is a Lehmer-Narkiewicz convolution. 
It may be noted that the multiplicativity preserving property of $\psi$ is not a necessary condition for the validity of (1.4). For example, if $T=$ $\left\{(1, n),(n, 1): n \in \mathbb{Z}^{+}\right\}$and $\psi(1, n)=\psi(n, 1)=n$ for all $n \in \mathbb{Z}^{+}$, then (1.4) holds trivially for all arithmetic functions $f$ with $f(1)=1$. However, $\psi$ does not preserve multiplicativity.

\section{References}

[1] E. CoHEN, Arithmetical functions associated with the unitary divisors of an integer. Math. Z. 74 (1960), 66-80.

[2] D. H. Lehmer, Arithmetic of double series. Trans. Amer. Math. Soc. 33 (1931), 945-957.

[3] W. NARKIEWICZ, On a class of arithmetical convolutions. Colloq. Math. 10 (1963), 81-94.

[4] V. Sitaramaiah, On the $\psi$-product of D. H. Lehmer. Indian J. Pure and Appl. Math. 16 (1985), 994-1008.

[5] V. SitARAMAIAH, On the existence of unity in Lehmer's $\psi$-product ring. Indian J. Pure and Appl. Math. 20 (1989), 1184-1190.

[6] V. Sitaramaiah, M. V. Subbarao, On a class of $\psi$-products preserving multiplicativity. Indian J. Pure and Appl. Math. 22 (1991), 819-832.

[7] V. Sitaramaiah, M. V. Subbarao, The identical equation in $\psi$-products. Proc. Amer. Math. Soc. 124 (1996), 361-369.

[8] V. Sitaramaiah, M. V. Subbarao, On regular $\psi$-convolutions. J. Indian Math. Soc. 64 (1997), 131-150.

[9] R. VAIDYAnAThasWAmy, The identical equation of the multiplicative functions. Bull. Amer. Math. Soc. 36 (1930), 762-772.

[10] R. VAIDyanathaswamy, The theory of multiplicative arithmetic functions. Trans. Amer. Math. Soc. 33 (1931), 579-662. (=[11], 326-414.)

[11] R. Vaidyanathaswamy, The collected papers of Prof. R. Vaidyanathaswamy. Madras University, 1957.

Jean-Louis Nicolas

Institut Girard Desargues

Mathématiques, Bât. 101

Université Claude Bernard (Lyon 1)

69622 Villeurbanne cédex, France.

E-mail : jlnicolaein2p3.fr

Varanasi SitaramaIAH

Department of Mathematics

Pondicherry Engineering College

Pondicherry 605014, India

E-mail : vsrmesatyam.net.in 